\title{
BMJ Visual analogue scales in stroke: what Open can they tell us about health-related quality of life?
}

\author{
Katerina Hilari, Lois-Danielle Boreham
}

To cite: Hilari K, Boreham L-D. Visual analogue scales in stroke: what can they tell us about health-related quality of life? BMJ Open 2013;3:e003309. doi:10.1136/bmjopen-2013003309

- Prepublication history for this paper is available online. To view these files please visit the journal online (http://dx.doi.org/10.1136/ bmjopen-2013-003309).

Received 28 May 2013 Revised 29 July 2013 Accepted 2 August 2013

Division of Language and Communication Science, City University London, London, UK

Correspondence to Dr Katerina Hilari; k.hilari@city.ac.uk

\section{ABSTRACT}

Objectives: Contrasting accounts exist on whether people with stroke are able to self-report on outcomes using visual analogue scales (VASs). We explored correlations between multi-item scale-rated health-related quality of life (HRQL) and VAS-rated HRQL after stroke, and compared those with versus without aphasia.

Design: Cross-sectional survey.

Setting: Community dwelling stroke patients living in London.

Participants: People with first stroke were recruited during their hospital stay and were assessed 3 months later.

Measures: The Frenchay Aphasia Screening Test, the Stroke and Aphasia Quality of Life Scale (SAQOL-39g) and a single vertical VAS.

Results: 73 people took part, 14 with aphasia. VAS scores were significantly correlated with the overall SAQOL-39g ( $r=0.69, p<0.01)$. SAQOL-39g subdomain scores were also correlated with VAS scores, with the psychosocial domain most highly correlated $(r=0.67$, $p<0.01)$ and the communication least correlated $(\rho=0.30$, $p<0.05)$. SAQOL-39g-VAS difference scores were higher for people with aphasia and the difference was significant (t $(71)=2.02, p<0.05)$.

Conclusions: Despite the significant correlation of the overall SAQOL-39g and the VAS-rated HRQL, subdomain results suggested that people considered mostly psychosocial aspects when rating their $\mathrm{HRQL}$ on a single VAS. Agreement was poorer for people with aphasia, raising issues for the use of VASs with people with aphasia.

\section{INTRODUCTION}

Health-related quality of life (HRQL) reflects the impact of a health state, here stroke, on a person's ability to lead a fulfilling life. It covers an individual's perception of and satisfaction with their physical, mental/emotional, family and social functioning. ${ }^{1}$ In stroke, an overarching rehabilitation aim is to improve each person's sense of well-being and quality of life. ${ }^{2}$

The simplest way to measure HRQL is through a single question or single-item measure. Such measures have an obvious

\section{ARTICLE SUMMARY}

Strengths and limitations of this study

- Study findings do not support the use of visual analogue scales as outcome measures for people with aphasia.

- Their scope is limited compared with multi-item measures.

- Of the 73 people who took part in the study, only 14 had aphasia. Findings need to be replicated in a larger study.

advantage: they are easy to administer and complete and thus less burdensome for clients and more efficient in terms of time and resources. One type of a single-item measure is a visual analogue scale (VAS) which typically comprises a $100 \mathrm{~mm}$ long line anchored at the ends with opposing states of a construct (eg, best possible and worst possible HRQL). In stroke, where people may have aphasia (difficulty communicating due to impaired understanding and use of language) or cognitive impairment, VASs seem particularly pertinent.

However, VASs and other single-item measures are unlikely to cover the broad scope of a complex construct, like HRQL; they may be interpreted in different ways by respondents; they cannot discriminate to a fine degree between different levels of an attribute and they tend to have limited reliability (prone to random error) as they do not produce consistent answers across time. ${ }^{3}{ }^{4}$ For these reasons, HRQL is typically measured with multi-item scales. Multi-item scales have more items which increase the scope of a measure; they are less open to variable interpretation; they are more precise and they are more reliable by allowing random errors to average out. ${ }^{34}$

In stroke, multi-item scales have been developed for the assessment of HRQL, yet only a few have been tested with people with aphasia. ${ }^{5-7}$ The Stroke and Aphasia Quality of Life scale (SAQOL-39g) has been adapted for use specifically with people with aphasia, 
with strong results on its reliability, validity and sensitivity to change in people with and without aphasia poststroke. ${ }^{6-8}$ It comprises 39 items covering physical, psychosocial and communication subdomains.

Despite the methodological limitations of VASs identified above, their apparent simplicity makes them attractive outcome measures for people with stroke. They have been successfully used to measure mood and self-esteem in stroke. ${ }^{9}{ }^{10}$ However, Pickard et al ${ }^{11}$ compared five different ways of measuring HRQL after stroke and found that a VAS was the most difficult to use without assistance and the least responsive to change.

This study had two aims (1) to explore how a single VAS on HRQL correlated with an established multi-item HRQL scale (SAQOL-39g) in people with stroke and (2) to see if there was a difference between people with aphasia and those without on agreement between VAS and SAQOL-39g scores.

\section{METHODS}

\section{Design and participants}

This study was part of a larger longitudinal study that assessed quality of life and emotional distress following stroke. $^{12}$ All participants gave written informed consent. Participants had to be: over the age of 18; admitted with a first stroke and requiring a stay of at least 3 days in the hospital. Exclusion criteria and full sample details are provided in the previous reports. ${ }^{12} 13$ For this study, participants were those of the original cohort $(n=87)$ who completed the required measures (see below) at 3 months poststroke $(n=73)$.

\section{Measures}

Participants completed the Frenchay Aphasia Screening Test $(\mathrm{FAST})^{14}$; the SAQOL-39g ${ }^{7}$ and a vertical VAS on their HRQL. The FAST is a screening tool to determine the presence of aphasia, with good evidence of reliability and validity. To take part in the study, people had to score a minimum of $7 / 15$ on the receptive domains of the FAST, to ensure that they could understand the questions of the SAQOL-39g ${ }^{7}$. The SAQOL-39g consists of 39 items that cover people's evaluation of their functioning in three domains: physical (eg, 'How much trouble did you have doing daily work around the house?'), psychosocial (eg, 'Did you feel discouraged about your future?') and communication (eg, 'How much trouble did you have getting other people to understand you?'). Overall and subdomain mean scores range from 1 to 5 ; higher scores indicate better HRQL. The VAS comprised a $100 \mathrm{~mm}$ vertical line anchored at the bottom by zero and the statement 'worst imaginable quality of life after stroke' and at the top by 100 and the statement 'best imaginable quality of life after stroke'. Participants were asked to look at the line and think about how their quality of life was affected by their stroke. There were small lines along the line at each centimetre interval and participants had to mark where they felt they were.

\section{RESULTS}

\section{Respondent characteristics}

Seventy-three people took part, 14 (19\%) were aphasic. Most $(88 \%)$ had an ischaemic stroke and most common type was partial anterior circulation stroke $(32 \%)$. The majority were white $(74 \%)$, men $(58 \%)$ and married/ had a partner $(55 \%)$. They ranged in age 18-91 (mean $(\mathrm{SD})=69.7(14))$.

\section{Correlations between SAQOL-39g scores and VAS ratings}

Table 1 presents scores on the VAS and the SAQOL-39g. They were normally distributed except for the SAQOL-39g communication subdomain. Comparing people with aphasia to those without, their scores were lower and more spread out both on the VAS (mean $(\mathrm{SD})=57.64$ (30.12) versus those without aphasia: 69.32 (21.98) ) and the SAQOL-39g (mean $(\mathrm{SD})=3.27(0.74)$ vs 3.97 (0.69) of those without aphasia) and its subdomains.

Table 2 and figures 1-3 present correlations between VAS ratings and SAQOL-39g scores for the overall sample and the two subgroups (aphasia vs no aphasia). Correlations between the VAS ratings and SAQOL-39g overall, psychosocial and physical domains were moderate-to-high $(\mathrm{r}=0.40-0.81)$ and significant (for 8/9 $\mathrm{p}<0.01)$. Despite this, the scatterplots reveal a more complex picture. Though they showed a positive somewhat linear trend, the points were quite scattered, especially on the VAS with SAQOL-39g physical domain, suggesting a weak association. There were also two outliers (from the subgroup of people with aphasia) who scored 0 on the VAS despite their scores on the SAQOL-39g. Correlations between the VAS and the SAQOL-39g communication subdomain were lower than for the other domains $(\rho=0.17-0.30 ; r=0.39)$. The scatterplots were quite spread out and as expected showed ceiling effects on the SAQOL-39 communication domain for the overall sample and those without aphasia.

\section{Comparisons between people with aphasia and those} without on agreement between VAS and SAQOL-39g scores

To see if there was a difference between people with aphasia and those without on agreement between SAQOL-39g and VAS, difference scores (SAQOL-39gVAS) were calculated, after converting VAS ratings and overall SAQOL-39g scores into standard scores. Difference scores (mean $(\mathrm{SD}))$ of aphasic participants $(-0.37(0.74))$ were greater than those of participants without aphasia $(0.10(0.79))$, and the difference was significant $(\mathrm{t}(71)=2.02, \mathrm{p}=0.047)$. The negative values of the scores of people with aphasia show they rated their HRQL higher with VAS than with SAQOL-39g. The opposite was true for those without aphasia.

\section{DISCUSSION}

The high and significant correlations between the SAQOL-39g and the VAS may seem to suggest that in a 
Table 1 Descriptive statistics of participants' scores on the VAS and the SAQOL-39g

\begin{tabular}{|c|c|c|c|c|c|}
\hline & \multirow[b]{2}{*}{ VAS } & \multicolumn{4}{|l|}{ SAQOL-39g } \\
\hline & & Overall & Physical & Psychosocial & Communication \\
\hline \multicolumn{6}{|l|}{ All $(n=73)$} \\
\hline Mean (SD) & $67.08(23.96)$ & $3.84(0.74)$ & $3.96(0.95)$ & $3.42(0.95)$ & $4.50(0.87)$ \\
\hline Median (IQR)* & & & & & $4.86(4.57-5.00)$ \\
\hline Minimum-maximum & $0-100$ & $2.18-4.97$ & $1.69-5.00$ & $1.56-5.00$ & $1.14-5.00$ \\
\hline \multicolumn{6}{|c|}{ Non-aphasic participants $(n=59)$} \\
\hline Mean (SD) & $69.32(21.98)$ & $3.97(.69)$ & $4.07(.88)$ & $3.54(.97)$ & $4.72(.52)$ \\
\hline Median (IQR)* & & & & & $4.86(4.71-5.00)$ \\
\hline Minimum-maximum & $25-100$ & $2.18-4.97$ & $1.69-5.00$ & $1.63-5.00$ & $2.43-5.00$ \\
\hline \multicolumn{6}{|c|}{ Aphasic participants $(n=14)$} \\
\hline Mean (SD) & $57.64(30.12)$ & $3.27(0.74)$ & $3.49(1.12)$ & $2.90(0.70)$ & $3.58(1.36)$ \\
\hline Minimum-maximum & $0-100$ & $2.24-4.69$ & $1.93-5.00$ & $1.56-4.21$ & $1.14-5.00$ \\
\hline
\end{tabular}

generic stroke sample a single VAS may yield similar findings on HRQL as a multi-item scale. However, the correlations between SAQOL-39g subdomains and the VAS showed that some aspects of those covered in the SAQOL-39g, that is, psychosocial, contributed more in the VAS ratings than others. Similar findings have also been reported by Pickard et $a l^{11}$ where, of five measures of HRQL, the VAS was most strongly associated with depression scores.

It is not surprising that in the overall sample the communication subdomain was least correlated with the VAS rating. The majority of the sample did not have aphasia and therefore communication would be expected to contribute less to HRQL than psychosocial and physical factors. However, for participants with aphasia, results were not as expected: VAS ratings were not significantly correlated with SAQOL-39g communication scores. This may suggest that people with aphasia were not thinking of communication when they rated their HRQL on a VAS. If this is the case, then VASs are less valid than multi-item scales in assessing HRQL. ${ }^{3}$ In a recent

Table 2 Correlations between VAS ratings and SAQOL-39g scores

\begin{tabular}{|c|c|c|c|}
\hline \multirow[b]{3}{*}{ SAQOL-39g } & \multicolumn{3}{|l|}{ VAS } \\
\hline & \multirow{2}{*}{$\begin{array}{l}\begin{array}{l}\text { Total } \\
\text { sample }\end{array} \\
\mathrm{N}=73\end{array}$} & \multicolumn{2}{|l|}{ By aphasia } \\
\hline & & $\begin{array}{l}\text { Non-aphasic } \\
n=59\end{array}$ & $\begin{array}{l}\text { Aphasic } \\
n=14\end{array}$ \\
\hline Overall & $0.69^{\star \star}$ & $0.64^{\star \star}$ & $0.81^{\star *}$ \\
\hline Physical & $0.52^{\star \star}$ & $0.40^{\star *}$ & $0.75^{\star \star}$ \\
\hline Psychosocial & $0.67^{\star \star}$ & $0.69^{\star \star}$ & $0.58^{*}$ \\
\hline Communication & $0.30^{*}$ & 0.17 & 0.39 \\
\hline \multicolumn{4}{|c|}{$\begin{array}{l}{ }^{*} p<0.05 . \\
{ }^{*} p<0.01 \text { level. } \\
\text { SAQOL-39g, Stroke and Aphasia Quality of Life Scale; VAS, } \\
\text { visual analogue scale. } \\
\text { Spearman's correlation co-efficient is represented by italic text; } \\
\text { Pearson's correlation co-efficient is represented by non-italic text. }\end{array}$} \\
\hline
\end{tabular}

systematic review of predictors of HRQL for people with aphasia, the importance of communication was highlighted, as communication disability was one of the strongest predictors. ${ }^{15}$ Our findings suggest that multiitem scales, such as the SAQOL-39g, prompt respondents to think about a wider variety of conributing factors to HRQL than a single VAS can do.

Looking at VAS_-SAQOL-39g agreement, people with aphasia had significantly lower agreement scores. They also used VASs differently to those without aphasia: they rated their HRQL more highly on a VAS, whereas those without aphasia rated their HRQL more highly on the SAQOL-39g. We explored whether other factors such as age and gender may have contributed to this difference, as, for example, older people may have greater difficulty in responding to VASs. ${ }^{16}$ We found no significant differences in demographic characteristics between people with aphasia and those without.

It is a limitation of the study that only 14 participants (19\%) had aphasia. Moreover, a fuller assessment of the sample's cognitive and communication deficits may have provided more information on how stroke survivors use VASs. These limitations notwithstanding, our findings have important clinical implications. In clinical practice, VASs are often used especially with people with aphasia, despite their limitations as assessments and outcome measures ${ }^{3}{ }^{4}$ because they are considered easy and accessible. This is problematic. VASs may provide a quick impression of HRQL but their scope is limited. Our study confirmed existing research that multi-item scales cover the broad construct of HRQL more comprehensively, whereas VASs reflect more psychosocial factors. ${ }^{11}$ Additionally, we showed that people with aphasia use VASs differently than other people with stroke; and had greater discrepancies between their VAS-rated and SAQOL-39g-rated HRQL. Valid and reliable multi-item scales should therefore be the tool of choice to assess HRQL in people with stroke and aphasia. 


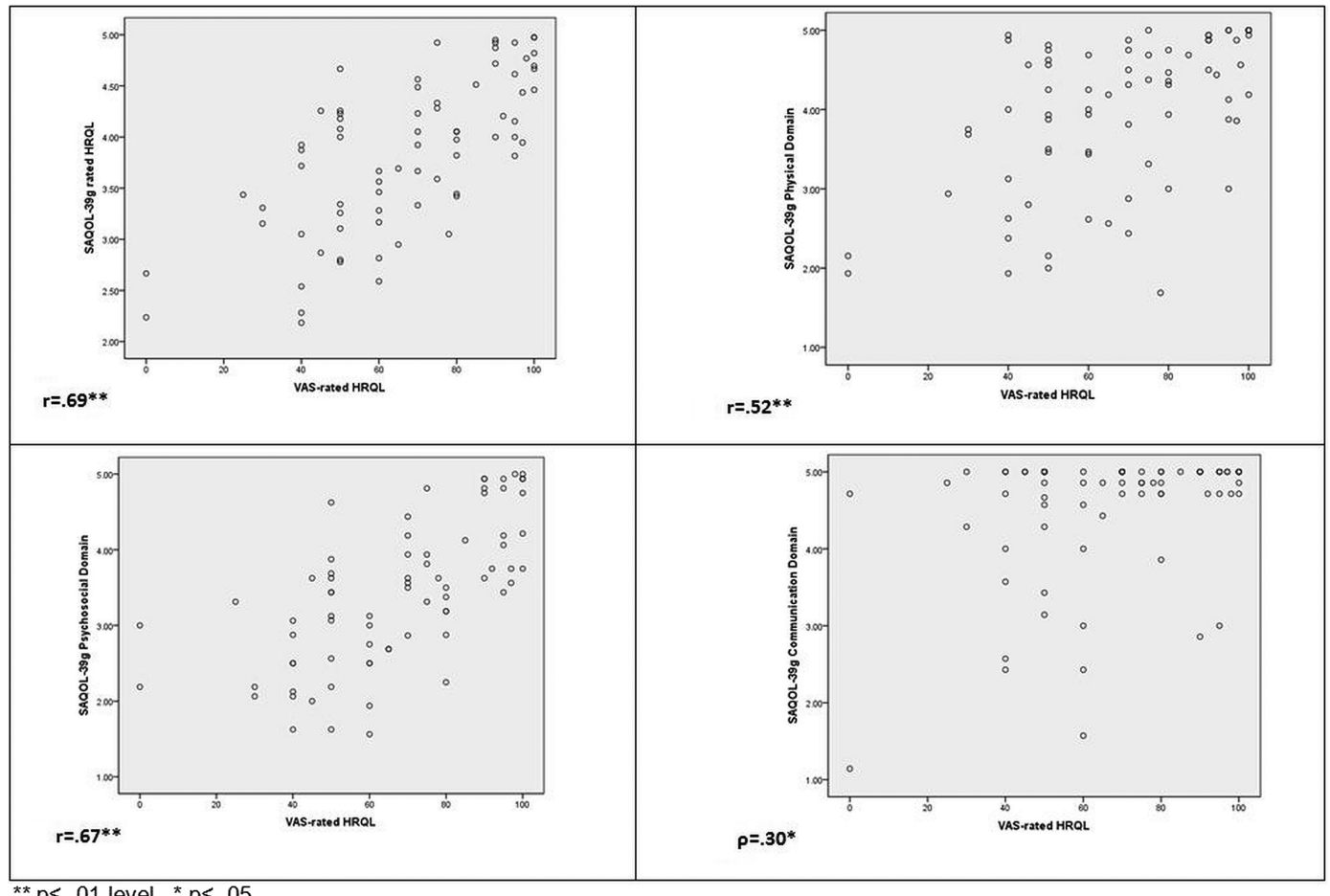

Figure 1 Total sample $(n=73)$ correlation scatterplots of Stroke and Aphasia Quality of Life Scale (SAQOL-39g) overall and subdomain scores and visual analogue scale (VAS)-rated health-related quality of life (HRQL) scores.

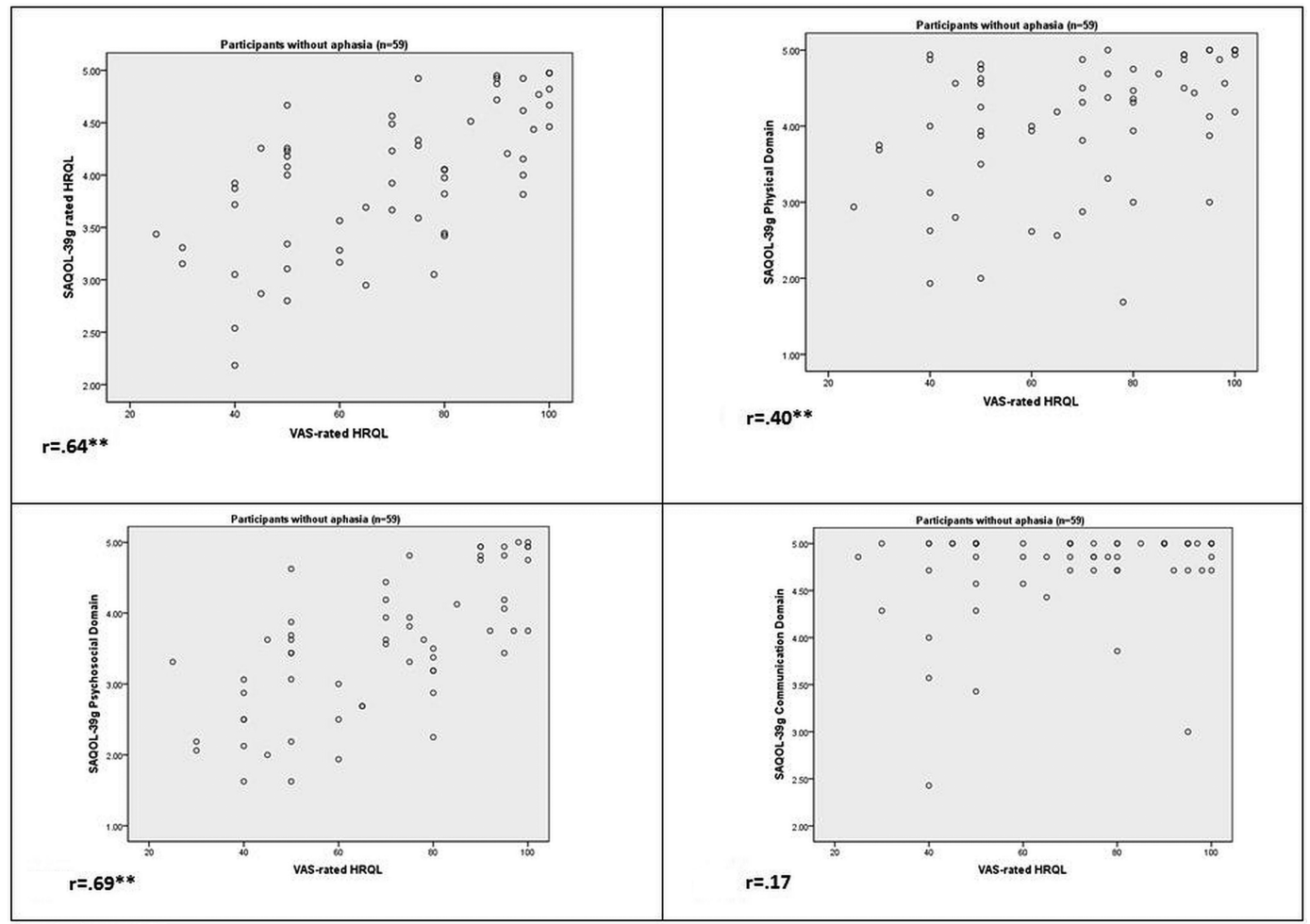

${ }^{*} p<.01$ level. ${ }^{*} p<.05$.

Figure 2 Participants without aphasia $(n=59)$ correlation scatterplots of Stroke and Aphasia Quality of Life Scale (SAQOL-39g) overall and subdomain scores and visual analogue scale (VAS)-rated health-related quality of life (HRQL) scores. 


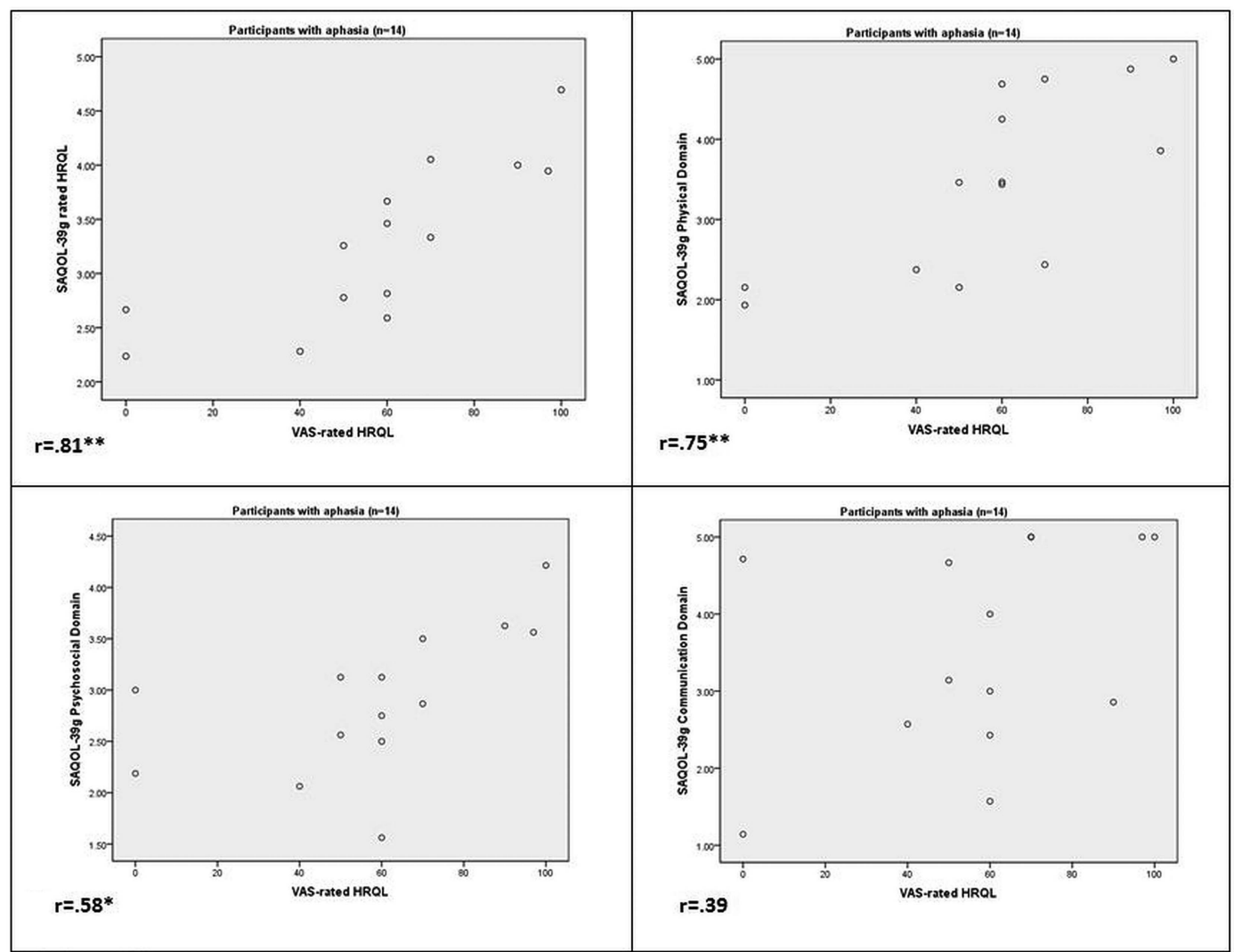

${ }^{* *} p<.01$ level. * $p<.05$

Figure 3 Participants with aphasia $(n=14)$ correlation scatterplots of Stroke and Aphasia Quality of Life Scale (SAQOL-39g) overall and subdomain scores and visual analogue scale (VAS)-rated health-related quality of life (HRQL) scores.

Acknowledgements The authors would like to thank all the participants and the clinical teams at St Mary's Hospital, London, and the Royal Free Hospital, London, without whom this project would not have been possible.

Contributors KH designed the study; collected the data; analysed and interpreted the data; wrote the paper and approved final version. L-DB contributed to data analysis; drafting of paper and approved the final version.

Funding This study was supported by a grant from the Consortium for Healthcare Research of the Health Foundation.

Competing interests None.

Patient consent Obtained.

Ethics approval St Mary's London NHS Trust and the Royal Free London NHS Trust.

Provenance and peer review Not commissioned; externally peer reviewed.

Data sharing statement No additional data are available.

Open Access This is an Open Access article distributed in accordance with the Creative Commons Attribution Non Commercial (CC BY-NC 3.0) license, which permits others to distribute, remix, adapt, build upon this work noncommercially, and license their derivative works on different terms, provided the original work is properly cited and the use is non-commercial. See: http:// creativecommons.org/licenses/by-nc/3.0/

\section{REFERENCES}

1. Berzon R, Hays RD, Shumaker SA. International use, application and performance of health-related quality of life instruments. Qual Life Res 1993;2:367-8.

2. Intercollegiate Stroke Working Party. National clinical guidelines for stroke. London: Royal College for Physicians, 2008.
3. Cano SJ, Hobart J. The problem with health measurement. Patient Prefer Adherence 2011;5:279-90.

4. Nunnally J. Psychometric theory. 2nd edn. New York: McGraw-Hill, 1978.

5. Doyle P, McNeil M, Hula W, et al. The burden of stroke scale (BOSS). Aphasiology 2003;17:291-304.

6. Hilari K, Byng S, Lamping D, et al. Stroke and Aphasia Quality of Life Scale (SAQOL-39): evaluation of acceptability, reliability, and validity. Stroke 2003;34:1944-50.

7. Hilari K, Lamping D, Smith $\mathrm{S}$, et al. Psychometric properties of the Stroke and Aphasia Quality of Life Scale (SAQOL-39) in a generic stroke population. Clin Rehabil 2009;23:544-57.

8. Caute A, Northcott S, Clarkson L, et al. Does mode of administration affect health-related quality-of-life outcomes after stroke? Int $J$ Speech Lang Pathol 2012;14:329-37.

9. Bennet $\mathrm{H}$, Thomas $\mathrm{S}$, Austen $\mathrm{R}$, et al. Validation of screening measures for assessing mood in stroke patients. Br J Clin Psychol 2006;45:367-76.

10. Brumfitt S, Sheeran P. The development and Validation of the Visual Analogue Self-Esteem Scale (VASES). Br J Clin Psychol 1999;38:387-400.

11. Pickard S, Johnson J, Feeny D. Responsiveness of generic healthrelated quality of life measures in stroke. Qual Life Res 2005;1:207-19.

12. Hilari K, Northcott S, Roy P, et al. Psychological distress after stroke and aphasia: the first six months. Clin Rehabil 2010;24:181-90.

13. Hilari K. The impact of stroke: are people with aphasia different to those without? Disabil Rehabil 2011:33:211-18.

14. Enderby P, Wood V, Wade D, et al. The Frenchay Aphasia Screening Test: a short, simple test for aphasia. Int Rehabil Med 1986;8:166-70.

15. Hilari K, Needle J, Harrison K. What are the important factors in health-related quality-of-life for people with aphasia? A systematic review. Arch Phys Med Rehabil 2012;93(S1):S86-95.

16. Radbruch L, Sabatowski R, Loick G, et al. Cognitive impairment and its influence on pain and symptom assessment in a palliative care unit: development of a minimal documentation system. Palliat Med 2000:14:266-76. 